\section{Pädiatrische Psychoonkologie}

\section{R. Zollinger}

\section{Einleitung}

Heute überleben aufgrund stetig verbesserter Therapiemethoden immer mehr Kinder ihre Krebskrankheit. Überleben heisst oft, über lange Zeit Therapien und Kontrollen über sich ergehen lassen zu müssen, Unsicherheiten angesichts eines möglichen letalen Verlaufs oder allfälliger Dauerschäden zu ertragen und über längere Zeit krank zu sein. Dies alles belastet nicht nur das Kind, sondern auch seine Familie und legt nebst der medizinisch-somatischen Betreuung eine Unterstützung im psychischen und sozialen Bereich nahe. So ist denn auch über all die Jahre eine weitgehende Zustimmung gewachsen, dass psychosoziale Betreuung zur ganzheitlichen Behandlung in der pädiatrischen Onkologie gehört, weil sie nötig und nützlich ist [1-4].

Die psychosoziale Betreuung umfasst psychologische, gesellschaftliche und materielle Aspekte. Sie unterstützt und ergänzt die somatische Behandlung.

\section{Psychoonkologische Arbeit}

Die Psychoonkologie deckt nicht alle, aber wichtige Aspekte bei der psychosozialen Betreuung ab. Sie sieht ihr Einsatzgebiet hauptsächlich bei folgenden Punkten:

- psychologische Exploration und Bearbeitung der Gesamtsituation unter Beachtung von psychosomatischen Phänomenen wie destruktiven Tendenzen usw.;

- psychologisches Bearbeiten von Lebensbedrohung, Todesnähe und Tod mit Patienten, Eltern und Geschwistern;

- Aufgreifen und Bearbeiten von Todesfallsituationen im Spital und deren Auswirkungen auf die anderen Patienten, Angehörigen und das Personal;

- Reflexion und Bearbeitung der Psychodynamik im Behandlungsteam, insbesondere auch der Themen Nähe/Distanz und eigene Betroffenheit angesichts Lebensbedrohung, Todesnähe und Tod;

- Zielerarbeitung und Rollenklärung im interdisziplinären Behandlungsteam.

Korrespondenz:

Dr. med. Ruedi Zollinger

Kinder- und Jugendpsychiatrische Dienste

Grossackerstrasse 7

CH-9000 St. Gallen
Ob und wie ausgedehnt diese Aspekte berücksichtigt werden können, hängt vom Ausbaustand der Psychoonkologie im Konzept der pädiatrischen Onkologie ab. Mit eine Rolle spielen Motivation und Bedürfnisse bei Patienten, Angehörigen und Fachpersonen. Je nachdem werden vor allem Kurzbesprechungen mit stützendem Charakter, Kriseninterventionen und knappe interdisziplinäre Rapporte durchgeführt oder es findet zusätzlich auch ein vertieftes Bearbeiten von verschiedenen Phänomenen mit Patienten, Angehörigen und Fachpersonen statt. Ein vertieftes Bearbeiten ist nicht immer möglich oder gewünscht. Es kann Verdrängung und andere zur Stabilisierung eingesetzte Abwehrmechanismen gefährden und belastend wirken. So können Eltern nach erfolgreicher, kurzzeitiger psychologischer Beratung weitere Kontakte mit dem Psychoonkologen ablehnen. Nur kurzzeitig gewünschte psychoonkologische Begleitung ist aber nicht nur unter dem Aspekt der Abwehr zu sehen. Bürgin [5] weist darauf hin, dass in vielen Fällen die Intervention des Psychotherapeuten nur für kurze Zeit gewünscht und nötig sei, besonders wenn die Eltern-Kind-Beziehung entwicklungsfähig genug ist. An Abwehrtendenzen im Pflegeteam ist zu denken, wenn beispielsweise auf den Tod einer zu Hause verstorbenen Patientin am Rapport nur indirekt eingegangen wird durch die Mitteilung, dass eine Mitarbeiterin später komme, weil sie an der Beerdigung eben dieser Patientin sei.

Immer wieder müssen sich psychoonkologisch Tätige fragen, ob mit Insistieren oder Gewähren den Betroffenen mehr gedient ist. Vorstellungen von Defusing [6] oder Debriefing* [9] können manchmal nicht oder nur minimal umgesetzt werden. Dies obschon wir um mögliche problematische Folgen bei Patienten, Angehörigen und Fachpersonen wissen, die über längere Zeit psychisch belastet worden sind.

Die Pflege der Kommunikation zwischen Fachpersonen einerseits, Patienten und Angehörigen andererseits und zwischen den verschiedenen betreuenden Berufsgruppen hat eine grosse Bedeutung und soll dem kommunikativen Chaos [10] entgegenwirken.

Die Stellung der psychoonkologischen Fachperson im Spitalteam muss erarbeitet und laufend geklärt werden. Welche Ziele werden angestrebt? Wird die Psychoonkologie als Konkurrenz empfunden? Wer ist zuständig für was?

\footnotetext{
* Das Debriefing ist eine Methode, welche strukturierte längere Gruppensitzungen umfasst. Personen, die ein Psychotrauma erlitten haben, rekonstruieren das Geschehene in der Gruppe. Es wird Raum geboten für die emotionale Verarbeitung der Situation. Das psychologische Debriefing stellt eine Möglichkeit der Prophylaxe dar, wenn verschiedene Voraussetzungen erfüllt sind, die zum "Critical Incident Stress Management" [7, 8] gehören. Das Defusing ist ein stützendes und verglichen mit dem Debriefing weniger formales Vorgehen.
} 
Für das Pflegeteam kann die psychoonkologische Fachperson eine Art Projektionsfläche sein. An ihr machen sich verständliche, aber wenig realistische Hoffnungen und Erwartungen nach rascher Lösung schwieriger Phänomene fest. Dies kann entlastend wirken. Regression und Projektion können Schutzbzw. Abwehrverhalten gegen Ängste sein und so im Rahmen institutionell verankerter Handlungs- und Beziehungsmuster der rationalen Arbeitsteilung, also dem Leistungszuwachs dienen [11].

Andererseits sieht sich die psychoonkologische Fachperson wiederholt mit Enttäuschungen über ihre Arbeit konfrontiert. Die Feststellung im Pflegeteam: "Der Psychoonkologe vollbringt keine Wunder" beinhaltet nebst Enttäuschung aber auch Entlastung und Eigenbestätigung: "Wir als Behandlungsteam machen es schon recht."

\section{Beispiele und Diskussion}

In den Kurzgesprächen mit Kindern hat der Psychoonkologe sogar "zwischen Tür und Angel» die Möglichkeit, stützende Bemerkungen zu machen und so entlastend zu wirken. Er lobt beispielsweise, wie geduldig und tapfer der Patient die Behandlung ertrage, obschon er an der Erkrankung ja nicht schuld sei. In dieser Formulierung ist die Stützung mit einem Hinweis auf mögliche Schuldgefühle kombiniert. Schliesslich wissen wir, dass das tumorkranke Kind die Krankheit oft als Bestrafung, die mit Schamgefühlen verbunden ist, empfindet. Je ernsthafter die Krankheit ist, desto mehr werden irrationale Vorstellungen ausgebildet [12].

Eine Jugendliche, der somatisch zwar Erleichterung, aber keine Heilung mehr verschafft werden konnte, wurde dem Psychoonkologen gemeldet, weil sie gespannt sei und verbalen Kontakt verweigere. Ihre Grosstante, für sie Mutterersatz, war eine häufige Besucherin, ihr verwitweter Vater war regelmässig, aber seltener am Krankenbett. Wie sich herausstellte, hatte sich die Situation zugespitzt, weil angesichts des nahen Todes im Gespräch mit der Patientin das Todesthema vermieden worden war. Die Angehörigen waren zwar seitens der Ärzte über den nahen Tod orientiert worden. Der Patientin gegenüber wurde aber Zuversicht vermittelt, von einem kommenden Ausflug ins Ausland gesprochen. Die psychologische Intervention bestand darin, dass der Psychoonkologe in Anwesenheit der Patientin zu Grosstante und Vater gesagt hat, es brauche sicher viel Kraft, von einer gemeinsamen Auslandsreise zu sprechen, wenn das Thema Abschied in der Luft liege. Ein kurzes Erschrecken bei der Grosstante über diese Äusserung wich bald einer Erleichterung, weil Sterben kein Tabuthema mehr war. Die Patientin selbst, die bislang mit geschlossenen Augen und trotz Ansprache stumm dalag, rief dem Psychoonkologen bei seinem Weggehen spontan einen Abschiedsgruss zu. Die Situation entspannte sich auf der psychischen Ebene. Erwartungsgemäss ist die Patientin leider wenige Tage später gestorben.
Die Mutter eines Knaben im Vorschulalter mit einem Tumorrezidiv zeigte sich so verzweifelt, dass das Abteilungsteam beschloss, die Mutter dem Psychoonkologen zuzuweisen. Sie war in verschiedener Hinsicht sehr belastet: ihr Mann hatte kein Berufseinkommen und litt unter psychischen Schwankungen. Die Mutter war die Erwerbstätige und konnte nur sehr bedingt vom Arbeitsplatz fernbleiben, um ihr krankes Kind zu besuchen. Speziell belastend für die Mutter war, dass sie sich in der ehelichen Partnerschaft nicht getragen fühlte. Insbesondere konnte sie sich mit ihrem Mann über den wahrscheinlichen Tod des Knaben nicht austauschen, da der Mann die Möglichkeit eines solchen Ausgangs leugnete und wahrscheinlich deshalb Kontakten mit Fachleuten wiederholt ausgewichen ist. Mit der Mutter wurden bis zum Tod des Kindes in grösseren Abständen längere stützende Gespräche geführt, für die sie dankbar war.

Die Mutter eines ungefähr 8jährigen Mädchens mit einer Hochrisikoleukämie war sehr niedergeschlagen. Dies angesichts der Schwere des Befundes. Dann aber auch deshalb, weil die Eltern eine konsequente schulmedizinische Behandlung gegen ärztlichen Rat lange hinausgezögert hatten. Die Stützung seitens des Psychoonkologen bestand hier unter anderem darin, dass er der Mutter für ihre langen Besuche im Spital nebst all ihren familiären Pflichten Anerkennung gezollt und seiner Überzeugung Ausdruck gegeben hat, dass sie so das Ihre zur Betreuung des Kindes beitragen könne.

Diese Beispiele illustrieren, wie wichtig Elternarbeit ist. Kazak et al. [13] untersuchten Eltern von überlebenden krebskranken Kindern und fanden, dass sowohl für Väter und Mütter Angst eine wichtige Ursache für spätere posttraumatische Stresssymptome war und nicht die objektiven medizinischen Daten. Sie folgerten, dass deshalb die Ängste der Eltern während der ganzen Behandlung und auch nach $\mathrm{Ab}$ schluss der Behandlung Beachtung brauchen. Dies kommt dann natürlich auch den Kindern zugute. Schliesslich kennen wir die Wechselwirkungen zwischen dem Befinden von Eltern und Kindern [14-16]. Laut Goldbeck [17] ergaben sich bei seiner Befragung von Eltern pädiatrisch/onkologischer Patienten Hinweise für ausgeprägte Wechselwirkungen zwischen dem Bewältigungsstil von Eltern und ihren Kindern. Sawyer et al. [18] schliessen aufgrund ihrer Untersuchungen, dass das Ausmass der Bedrängnis von Müttern nach der Krebsdiagnose bei ihrem Kind einen wichtigen Einfluss auf die spätere psychologische Einstellung des Kindes haben kann. Kusch et al. [19] fanden, dass höher belastete Eltern eine ungünstige Auseinandersetzung mit der Möglichkeit einer Krebserkrankung aufweisen. Sie würden mit der Diagnosestellung weniger Emotionen stattdessen vermehrt negative Bemerkungen verbinden. Sie hätten deutlich mehr Gefühle eigener Schuld an der Erkrankung des Kindes, würden jedoch die Angebote sozialer Unterstützung häufiger ablehnen als die weniger belasteten Eltern. 


\section{Literatur}

1 Zünd M (hrsg. von Herzka HS). Betreuung krebskranker Kinder. Zürich: pro juventute; 1993.

2 Häberle H, Schwarz R, Brandeis W. Psychosoziale Versorgung der onkologischen Kinderstation. Der Kinderarzt 1988: 4:462-7.

3 Topf RJ. Medizinische Krebsaufklärung als psychosoziale Notwendigkeit. Kinderanalyse 1997;5(3):285-311.

4 Thiel R. Entwicklung, Struktur und Auswertung psychosozialer Betreuung krebskranker Kinder und ihrer Familie. Hamburg, Dissertation; 1990.

5 Bürgin D. Begleitung sterbender Kinder. In: Rösing I, Petzold H (Hrsg.). Die Begleitung Sterbender. 2. Auflage. Paderborn: Junfermann; 1992. S. 377-90.

6 Morrissey M. Counselors "helping the helpers" from becoming casualties. Counseling Today 1994;37(6):1, 6, 7, 12.

7 Mitchell JT, Everley GS. Critical incident stress management and critical incident stress debriefings: evolution, effects and outcomes. In: Raphael B, Wilson JP (eds.). Psychological Debriefing. Cambridge: University Press; 2000. p. 17-90.

8 Perren-Klingler G. Präventive Massnahmen zur Erhaltung der psychischen Gesundheit. Informationsschrift KSD 1999; 1:30-3.

9 Robinson RC, Mitchell JT. Evaluation of psychological debriefings. J Traum Stress 1993;6(3):367-82.

10 Topf R, Trimmel J, Vachalek L, Felsberger C, Gadner H. Das psychosoziale Betreuungskonzept der pädiatrischen Onkologie des St.-Anna-Kinderspitals Wien. Paediatr Paedol 1997;32:13-23.
11 Mentzos S. Interpersonale und institutionalisierte Abwehr. Frankfurt am Main: Suhrkamp Taschenbuch; 1988.

12 Bürgin D. Kinderpsychiatrie und lebensbedrohende Krankheit. Schweiz Ärztezeitung 1980;61(6):313-21.

13 Kazak AE, Stuber ML, Barakat LP, Meeske K, Guthrie D, Meadows AT. Predicting posttraumatic stress symptoms in mothers and fathers of survivors of childhood cancer. J Am Acad Child Adolesc Psychiatry 1998;37:823-31.

14 Bürgin D. Zur Prävention psychischer Störungen nach dem Verlust eines Geschwisters im Kindesalter. Sozial- und Präventivmedizin 1977;22:46-52.

15 Harder GM (hrsg. von Herzka HS). Sterben und Tod eines Geschwisters. 2. Auflage. Zürich: pro juventute; 1992.

16 Zollinger R. Psychotherapie eines Knaben mit einer Depression nach dem Tod von zwei seiner Brüder. Prax Kinderpsychol Kinderpsychiat 1997;46:727-31.

17 Goldbeck L. Die familiäre Bewältigung einer Krebserkrankung im Kindes- und Jugendalter. Prax Kinderpsychol Kinderpsychiat 1998;47:552-73.

18 Sawyer MG, Streiner DL, Antoniou G, Toogood I, Rice M. Influence of paternal and family adjustment on the later psychological adjustment of children treated for cancer. J Am Acad Child Adolesc Psychiatry 1998;37:815-22.

19 Kusch M, Labouvie H, Bode U. Unterschiede im aktuellen Befinden zu Beginn der Krebstherapie und möglichen Folgen: eine Pilotstudie in der pädiatrischen Onkologie. Prax Kinderpsychol Kinderpsychiat 1994;43:98-106. 